\title{
2019 Travel Grant Recipients
}

$\mathrm{T}$ o increase participation in the Annual Meeting, the association awarded travel grants to graduate students, scholars, and special program participants for the 2019 Annual Meeting in Washington, DC. The travel grants subsidize the cost of travelling to the meeting. Every year, Cambridge University Press provides a generous

TRAVEL GRANT RECIPIENTS

Rania Abdelnaeem

Muhammad Hassan Bin Afzal

Rebeca Agosto Rosa

Zainab Alam

Cagil Albayrak

Karen Albert

Garrett Albistegui Adler

Marco Alcocer

Raul Aldaz

Noaman Ali

Luai Allarakia

Safa Al-Saeedi

Ugur Altundal

Bulat Akhmetkarimov

Engi Amin

Eran Amsalem

Jingjing An

Jessica Andersson-Hudson

Merih Angin

Joseph Anthony

Carlos Antonio de Oliveira

Reiko Arami

Ayca Arkilic

Selim Erdem Aytac

Sumitra Badrinathan

Elizabeth Baisley

Samuel Baltz

Bree Bang-Jensen

Syahar Banu

Genevieve Bates

Polina Beliakova

Sara Belligoni

Raphael BenLevi

Matthew Bergman

Nicolas Bichay

Reuben Binns

Christine Bird

Jason Blessing

Douglas Block

Marco Bocchese

Tabitha Bonilla

Joshua Boston

Aimée Bourassa

Peter Bowman

Tristan Bradshaw

Shawna Brandle

Caroline Brandt
Christine Bricker

Evelyne Brie

Margaret Brower

Kirill Bryanov

Jordan Butcher

Beyza Buyuker

Devon Cantwell

Paloma Caravantes-Gonzalez

Vanessa Carrion Yaguana

Fatih Cetin

Nathan Chan

Zenobia Chan

Jung-Chun Chang

Danielle Charette

Andrew Chater

Kerry Chavez

Gabrielle Cheung

Alexandra Chinchilla

Yvonne Chiu

Vladimir Chlouba

William Christiansen

Paula Clerici

Clayton Cleveland

Geneva Cole

Jessica Collier

Claudia Roberta Combei

Nadege Compaore

Brendan Cooley

Charles Crabtree

Paolo Crivellari

John Crowell

Santiago Cunial

Karol Czuba

Debak Das

Caitlin Davies

Mary Jane Dempsey

Hari Dhakal

Gustavo Diaz

Lora DiBlasi

Alona Dolinsky

Meredith Dost

Sagnik Dutta

Rotem Dvir

Christoph Dworschak

Mireille Manga Edimo

Jessica Edry

Karim Elkady

John Emery

Cengiz Erisen

Elif Erisen sponsorship to graduate students. APSA was glad to welcome the following graduate student attendees, scholars, and special program participants to the 2019 Annual Meeting. This list is not exhaustive of all travel grants given by APSA:
Ugurcan Evci

Guy Eyre

Judit Fabian

Sandor Fabian

Rodrigo Fagundes Cezar

Mona Farag

Claudia Faria

Asif Farooq

Christopher Faulkner

Jeff Feng

Ana Luiza Ferreira

Paul Friesen

Lillian Frost

Chengyu Fu

Matteo Fumagalli

Miguel Garza Casado

Michael Gavin

Federica Genovese

Matthew Geras

Matteo Gigliolo

Shauna Gillooly

Burak Giray

Amélie Godefroidt

Stephen Goggin

Katherine Goktepe

Juan Gomez-Cruces

Gabriel Goodliffe

Mary Gorham

Erica Gould

Tanushree Goyal

Rohan Gudibande

Catherine Guisan

Kristina Gushchina

Signy Gutnick Allen

Nicholas Haas

David Hageboelling

Jamie Hagen

Brian Hamel

Soha Hammam

Jordan Hamzawi

Kangwook Han

Alex Haskins

Miaad Hassan

Matthew Hauenstein

Ron Hayduk

Alexander Held

Kevin Henderson

Bastian Herre

Aubree Herrin

Federico Holm 
Parakh Hoon

Adil Hossain

Juhi Huda

Annabelle Hutchinson

Gennadii Iakovlev

Alice Iannantuoni

Ulkar Imamverdiyeva

Nagisa Ishinabe

Brian Jabarian

Nicholas Jacobs

Anton Jager

Anne Jamison

Hye Ryeon Jang

Andrew Janusz

Athmeya Jayaram

Laura Jenkins

Veronique Jerome

Xinhui Jiang

Philip Johnson

Michael Jones

Melina Juarez

Courtney Juelich

Yujin Jung

Sebastian Jungkunz

Tatjana Jungkunz

Agabek Kabdullin

Jose Kaire

Joseph Kaminski

Stephanie Kang

Wynand Kastart

Sean Kates

Ruchan Kaya

Franziska Keller

John Kenny

Ceren Keser

Namhoon Ki

Kimberly Killen

Daegyeong Kim

Hannah Kim

Hansaem Kim

Hyunwoo Kim

Minju Kim

Seonhee Kim

Young Mi Kim

Denys Kiryukhin

Korhan Kocak

Melanie Kolbe

Mzilikazi Kone

Nazli Konya

Kate Korycki

Svetlana Kosterina

Matthias Kroenke

Max Kuhelj Bugaric

Sidita Kushi

Suyatno Ladiqi

Julius Lagodny

Juan Larrosa-Fuentes

Julia Lau

Isabelle Laurenzi

Katharina Lawall
Alexandra Lazar

Catarina Leão

Adele Lebano

Bomi Lee

Danbee Lee

Helen Lee

Kyu Young Lee

Myunghee Lee

Shinkyu Lee

Youngchae Lee

Todd Lehmann

Theresa Leimpek

Tara Lennon

Alexis Lerner

Michael Lerner

Vivien Leung

Jia Li

Jingwei Li

Siyao Li

Yimeng Li

Ying Li

Zhengyan Li

Rodrigo Lins

Ryan Yu-Lin Liou

Dongshu Liu

Shiyao Liu

Maria Livaudais

Jaime Lluch

Lucie Lu

Caleb Lucas

Kristin Lunz Trujillo

Gregory Lyon

Yoshira Macias-Mejia

Janica Magat

Beatrice Magistro

Cameron Mailhot

Rima Majed

Pero Maldini

Deepak Malghan

Elliot Mamet

Summer Marion

Brendan Skip Mark

Yvonni Markaki

James Martherus

Daniella Mascarenhas

Takuya Matsuda

Miku Matsunaga

Ilya Matveev

Barry Maydom

Sebastian Mayer

Kyong Mazzaro

John McAndrews

Stefan McCabe

Martin McCallum

Caitlin McMullin

Heather McCambly

Charles McClean

Josh McCrain

Sultan Mehmood

Anna Meier
Claudio Arturo Méndez

Mary Anne Mendoza

Anil Menon

Luca Messerschmidt

Shawna Metzger

Eli Meyerhoff

Anwar Mhajne

Carla Michalak

Matthew Millard

Austin Mitchell

Yazmin Morlet Corti

Valentina Moro

Louis-Philippe Morneau

Eric Mosinger

Alexander Motchoulski

Taishi Muraoka

Rachel Myrick

Gabriel Nahmias

Nicholas Napolio

Shaheen Naseer

Rita Nassar

Grace Natusch

Pedro Neiva

Austin Nelson

Rebecca Nielsen

Emeka Thaddues Njoku

William Nomikos

Kelsey Norman

Jacob Nyrup

Neil O'Brian

Shannon O'Brien

Nina Obermeier

Anastassia Obydenkova

Marzia Oceno

Soomin Oh

Janet Oluwaleye

Jennifer Oser

Aykut Ozturk

Ju Yeon Park

Ioana Panaitiu

Anu Pauliina Patana

Reid Pauly

Miguel Pereira

Keston Perry

Alexandra Petrachkova

Katharine Petrich

Ariel Petrovics

David Peyton

Kyle Peyton

Jonathan Phillips

Samuel Piccolo

Patrick Pierson

Barbara Piotrowska

Alesha Porisky

Evgeny Postnikov

Bhumi Purohit

Dan Qi

Jing Qian

Daniel Quiroga-Angel

David Ragazzoni 
Nahrain Rasho

Eli Rau

Tanika Raychaudhuri

Frank Reichert

Jiacheng Ren

Tyler Reny

Mara Revkin

Catherine Reyes-Housholder

Hannah Ridge

Karin Riedl

Viviana Rivera-Burgos

Corey Robin

Kaitlyn Robinson

Vinicius Rodrigues Vieira

Kathleen Rogers

Jennifer Rogla

F Benjamin Rosche

Emma Rosenberg

Sohyeon Ryu

Eduardo Salinas

Luke Sanford

Maria Paula Saffon Sanin

Gustavo Santos

Gregory Sasso

Yuko Sato

Dean Schafer

Luis Schenoni

Alain Schläpfer

Marcel Schliebs

Eric Schmidt

Jessica Schoenherr

Rachel Schoner

Anne Louise Schotel

Avshalom Schwartz

Emily K M Scott

Miklos Sebok

Karen Sebold

Kaitlin Senk

Min Hee Seo

Yixue Shao

Sally Sharif

Svitlana Shcherbak

Carlos Shenga
Sujeong Shim

Hyo-Won Shin

Mi Jeong Shin

Aditi Shirodkar

Khushi Singh Rathore

Jessica Soedirgo

Kailash Srinivasan

Mette Marie Staehr Harder

Kai Stern

Anette Stimmer

Andrew Stone

Ella Street

Mary Stylidi

Kyungwon Suh

Jessica Sun

Meicen Sun

Clara Suong

Jacob Swanson

Christine Sylvester

Georgiy Syunyaev

John Taden

Halit Tagma

Yurina Takeichi

Honggang Tan

Chengzuo Tang

Basak Taraktas

Nathan Tarr

Ari Baghdassar Tatian

Tatum Taylor

Chris Tenove

Andrew Thompson

Oskar Timo Thoms

Patrick Tiney

Lev Topor

Mart Trasberg

Minh Trinh

Federico Trombetta

Eteri Tsintsadze-Maass

Hans Tung

Linnea Turco

Amilee Turner

Jacob Turner

Matthew Uttermark
Jimena Valdez

Emily Van Duyn

Saskia Van Wees

Clara Vandeweerdt

Fabricio Vasselai

Vania Ximena Velasco Guachalla

Peter Verovsek

Paola Vesco

Danielle Villa

Sasha Volodarsky

Emily Wager

Gauri Wagle

Gang Wang

Yaping Wang

Shana Warren

Yao Wen

Zhuoyi Wen

Crystal Whetstone

Rob Williams

Hannah Wilson

Marta Wojciechowska

Brian Wolfel

Mark Wolfgram

Fuzuo Wu

Kuan-Sheng Wu

Nicole Wu

Patrick Wu

Yan $\mathrm{Xu}$

Nicole Yadon

Şebnem Yardımcı-Geyikçi

Luwei Ying

Jesse Yoder

Jongeun You

Basileus Zeno

Ruodan Zhang

Zhu Zhang

Bi Zhao

Yangzi Zhao

Bang Zheng

Eva Ziegler

Justin Zimmerman

Samantha Zuhlke

Kelebogile Zvobgo 